\title{
Laparoscopic omental flap for the treatment of major sternal wound infection after cardiac surgery
}

\author{
Francesco Puma, MDa \\ Costanzo Fedeli, $\mathrm{MD}^{\mathrm{b}}$ \\ Paolo Ottavi, MDa \\ Giuseppina Porcaro, MDa \\ Giovanni Battista Fonsi, $\mathrm{MD}^{\mathrm{a}}$ \\ Alessandro Pardini, MD ${ }^{\mathrm{a}}$ \\ Giuliano Daddi, MD
}

From the Department of Thoracic Surgery, Terni, Italy, ${ }^{\mathrm{a}}$ and Department of General Thoracic Surgery, University of Perugia Medical School, Perugia, Italy. ${ }^{\mathrm{b}}$

Received for publication Nov 19, 2002; revisions requested Jan 21, 2003; revisions received Feb 4, 2003; accepted for publication March 6, 2003.

Address for reprints: Francesco Puma, MD, Chirurgia Toracica, Ospedale Civile S. Maria, 05100, Terni, Italy (E-mail: francescopuma@aospterni.it).

J Thorac Cardiovasc Surg 2003;126: 1998-2002

Copyright () 2003 by The American Association for Thoracic Surgery

$0022-5223 / 2003 \$ 30.00+0$

doi:10.1016/S0022-5223(03)00709-8
Background: The ideal reconstructive procedure after sternal debridement is still a matter of debate. The omentum might be theoretically preferable for its favorable properties, but it is seldom used because it entails the added trauma of a laparotomy.

Methods: Three female patients with severe osteomyelitis after myocardial revascularization underwent sternal debridement and filling of the defect with a laparoscopically prepared omental flap. Sternal wound closure was achieved as a singlestage procedure in 2 patients. The third patient had a poststernotomy septicemia and required a 2-stage procedure. The abdominal procedures were conducted through 3 operating 5-mm ports. Omental flaps were developed by complete separation from the transverse colon and lengthening by division of some anastomosing arteries between gastroepiploic vessels and Barkow's arcade. Thoracic transposition of the omentum was achieved through a 5-cm diaphragmatic incision. The flaps were able to reach the base of the neck and fill the sternal defect in all patients.

Results: A smooth postoperative course was observed. Oral intake was started from day 2; sole oral nutrition was maintained from day 3 or 4 . Optimal wound healing was observed with minimal or absent local discomfort. Minor transient paradoxical movements of the anterior chest wall disappeared within 1 month. Postoperative hospital stay was 9,14 , and 14 days, respectively.

Conclusions: Laparoscopic omentoplasty, compared with the open procedure, entails several advantages for the treatment of sternal osteomyelitis. The introduction of minimally invasive techniques may widen the indications for the use of the omentum in the treatment of major sternal wound infections.

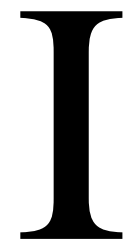

nfection of a median sternotomy wound is a dreaded and sometimes devastating complication in cardiac surgery. The incidence rate ranges from $0.4 \%$ to $6.9 \%$ and mortality ranges from $7 \%$ to $80 \%,{ }^{1}$ depending on the severity of the infection, the treatment, and the patient's general condition.

Incidence rates and mortality do not show a significant decrease in the last decade, ${ }^{2,3}$ which is likely due to coronary artery bypass grafting $(\mathrm{CABG})$ also being performed more commonly on older and more critically ill patients who were previously excluded from such a procedure.

The treatment of complex wounds requires total removal of the infected bone (and costal cartilages, when appropriate). Second, a well-vascularized replacement tissue must be found. The ideal reconstructive procedure is still a matter of debate. ${ }^{2}$ The omentum might be theoretically preferable for its favorable properties, ${ }^{4}$ but it is seldom used because of 2 major disadvantages: (1) it entails heavier surgical trauma and (2) the procedure puts a sterile cavity in contact with a contaminated 
field. Therefore, the muscle flap closure is generally preferred, basically to reduce the surgical trauma. ${ }^{3}$

We have treated several patients by omental flap transposition through a traditional laparotomy. This report presents the successful management of severely infected median sternotomy wounds by sternal debridement and reconstruction with a laparoscopically prepared omental flap.

\section{Materials and Methods Patients}

Three female patients (median age 64 years) were referred to our institution for severe sternal osteomyelitis after myocardial revascularization performed 23, 40, and 190 days earlier. The observed risk factors for sternal osteomyelitis were as follows: insulindependent diabetes mellitus, hypertension, and left internal mammary artery harvesting for the CABG (all patients); history of smoking ( 2 patients); use of intraaortic balloon pump, obesity, and reexploration of the sternotomy wound for hemorrhage (1 patient, respectively). Only 1 patient had been submitted to CABG in our hospital.

When initially admitted to our department, 2 patients were in very poor general conditions with sepsis; computed tomography scan demonstrated concomitant mediastinitis and bilateral pleural effusions. The third patient had a chronic, oligosymptomatic, sternal dehiscence. Sternal wound infections were classified according to Jones and associates ${ }^{3}$ : type $2 \mathrm{~b}$, exposed bone, unstable wired sternotomy; type $3 \mathrm{a}$, exposed fractured bone, exposed heart; type $3 \mathrm{~b}$, type $2 \mathrm{~b}$ with septicemia. Two patients had a documented staphylococcal infection of the wound: Staphylococcus aureus, epidermidis, and chromogenes. In the third patient, Pseudomonas aeruginosa was identified from the wound and coagulase-negative staphylococcus from the blood cultures. Intravenous antibiotic therapy was instituted according to the cultures' sensitivity.

Wound closure was achieved as a single-stage procedure in 2 patients. The patient with septicemia was treated with a 2-stage procedure: an urgent radical debridement was performed under general anesthesia, associated with bilateral chest drainage. Wide opening of all mediastinal abscess cavities was performed; the left side of the wound appeared to be more necrotic compared with the right side. Removal of the whole soft and necrotic left hemisternum together with the affected left costal cartilages was carried out. The right hemisternum was only debrided until solid and bleeding bone tissue was found. Bone cultures were taken at this level. A significant improvement of the clinical conditions was obtained after debridement so that 8 days later the patient underwent the reconstructive procedure.

\section{Technique of Laparoscopic Thoracic Transposition of the Greater Omentum}

The patients were intubated with a single lumen endotracheal tube and placed in the supine position with both arms adducted. An inflating bag was positioned under the shoulders. A 10-mm Hasson trocar was placed through a $2-\mathrm{cm}$ midline vertical incision, just above the umbilicus, and a $30^{\circ}$ telescope was inserted; the pneumoperitoneum was then started. Figure 1 illustrates the trocar's placement.

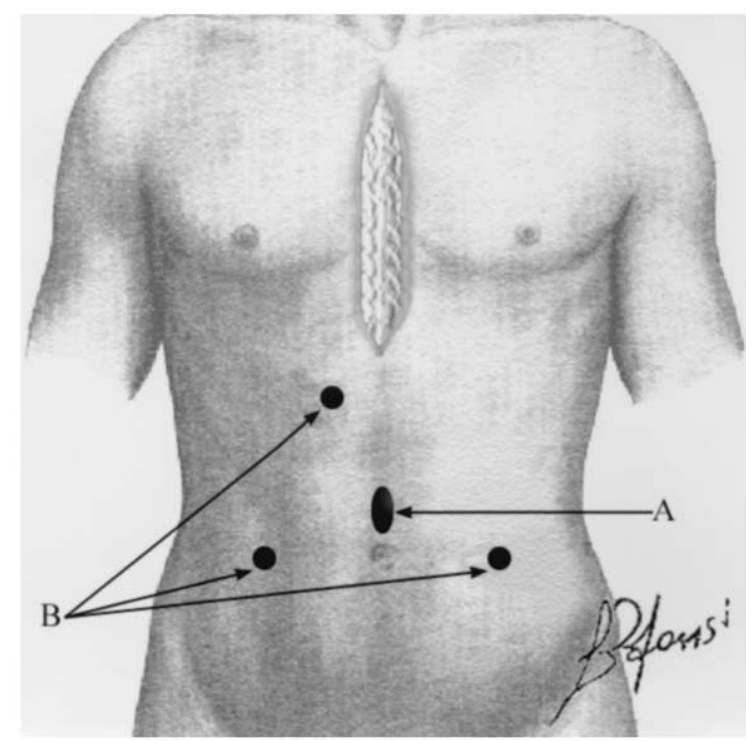

Figure 1. Port placement for laparoscopic omentoplasty. A, Tenmillimeter trocar $\left(30^{\circ}\right.$ angled telescope). B, Five-millimeter trocars (operating ports).

In 2 patients we found adhesions related to previous abdominal surgery (appendectomy in 1 patient; abdominal hysterectomy and cholecystectomy in the other). Adhesions were divided with ultrasound scissors (Harmonic Scalpel; UltraCision: Ethicon EndoSurgery, Inc, Cincinnati, Ohio).

Complete separation of the omentum from the transverse colon was carried out. The colon was held and pulled caudally with atraumatic forceps through 1 of the inferior lateral ports. The ultrasound scissors were inserted through the contralateral port and separation was performed on the anatomic avascular plane. The superior port was used to grasp the gastric wall to facilitate the dissection. The right gastroepiploic artery (dominant blood supply) was left untouched and the omental flap developed at the expense of Barkow's arcade, dividing some of the anastomosing arteries (anterior and left; Figure 2). Thoracic transposition of the mobilized omentum was achieved through a $5-\mathrm{cm}$ substernal diaphragmatic incision. The omentum was grasped with standard Duval forceps, gently pulled out from the abdominal cavity, and delivered into the mediastinal defect.

Great care was used to control the correct position of the stomach greater curvature, which could have been stretched cranially during the omental transposition.

Complete wound debridement followed thoracic omental transposition in the 2 patients submitted to a single-stage closure. The flap provided enough bulk to fill the space. In 1 patient, the bulk was even excessive at the lower portion of the wound, and it was necessary to reduce it by peripheral resections to facilitate skin closure without tension and to avoid heart compression.

The omental flap was fixed with interrupted sutures to the base of the neck, to the lateral edges of the defect, and to the margins of the diaphragmatic opening. The trocars were removed under visual control and the abdominal wall repaired with synthetic reabsorbable interrupted sutures. Two silicone drains were posi- 


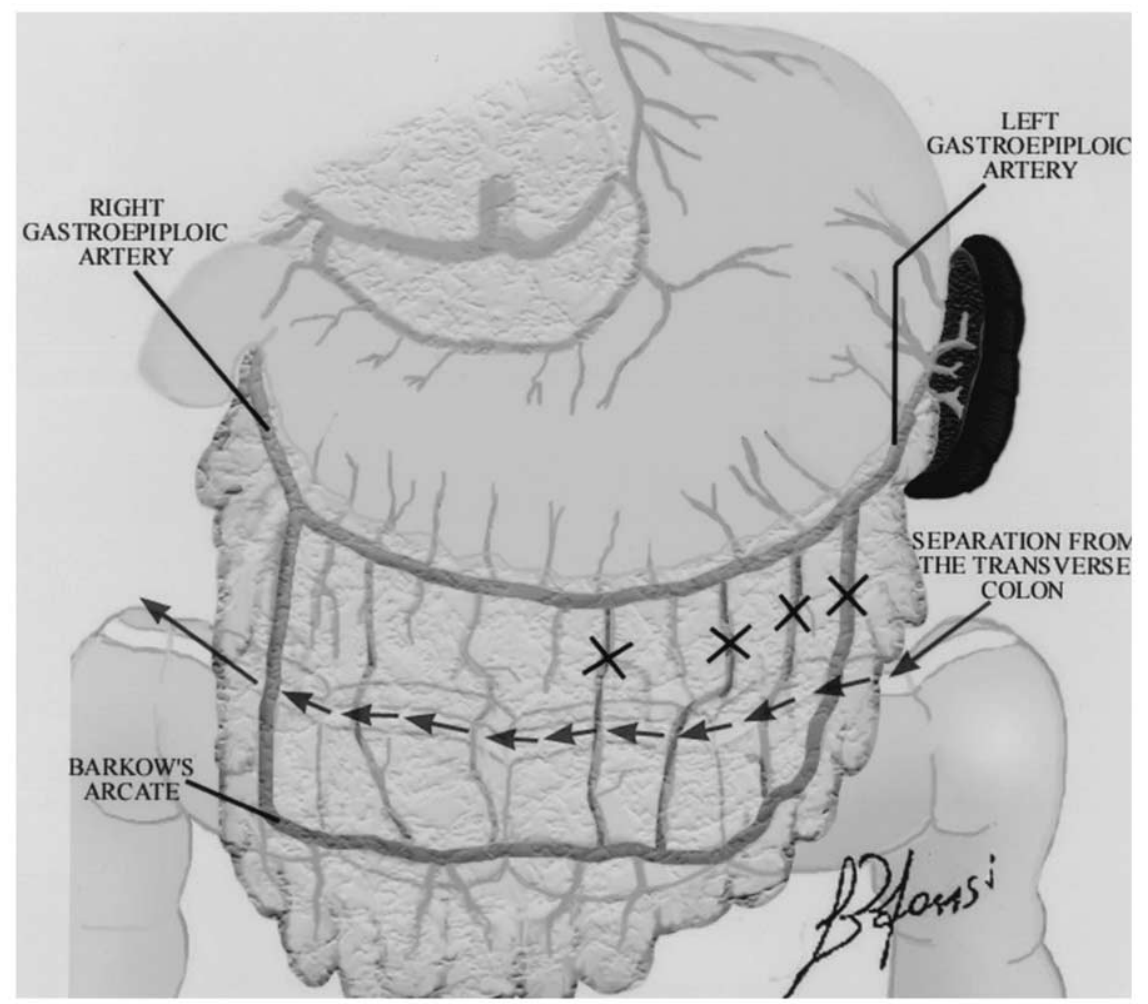

Figure 2. Technique of laparoscopic omental flap, with complete separation of the omentum from the transverse colon and lengthening of the mobilized omentum by division of some anastomosing arteries between gastroepiploic vessels and Barkow's arcade.

tioned, 1 beneath and 1 over the omental flap. One such thoracic drain was inserted through the skin incision created for the superior abdominal trocar. No abdominal drains were used. A nasogastric tube was left in place.

In the septic patient showing severe preoperative anemia, 2 blood transfusions were administered.

\section{Results}

The duration of the laparoscopic procedures was 65, 75, and 90 minutes, respectively. The patients recovered well from general anesthesia and resumed spontaneous breathing promptly.

Postoperative course was smooth in all patients; a mild febrile response of short duration (respectively 2, 3, and 5 days) was observed. Effective intestinal peristalsis resumed immediately and the nasogastric tube was removed on day 1. Oral intake was started from day 2; sole oral nutrition was maintained from day 3 or 4 . The central venous catheters were removed on days 8,10 , and 12 , respectively.

Optimal wound healing was observed with minimal or no local discomfort. Minor paradoxical movements of the anterior chest wall were observed in 2 patients. The median postoperative hospital stay was 14 days (range 9-14 days).
All patients continued to receive home intravenous antibiotic therapy for 4 weeks.

Outpatient follow-up (from 20 to 45 days) showed dramatic improvement of the patients' general conditions, optimal wound healing, and resolution of chest wall paradoxical movements; blood tests demonstrated normal glucose values, normal leukocyte count, and significant decrease of the erythrocyte sedimentation rate.

\section{Discussion}

Infection of the median sternotomy wound is a not an uncommon complication after cardiac surgery (roughly $1 \%$ incidence rate at our institution). The clinical importance of such a problem varies from an easily treatable superficial abscess to life-threatening conditions, such as suppurative mediastinitis, especially if associated with septicemia.

Complex median sternotomy wounds may be difficult to manage, basically for 3 factors:

1. The severity of the condition. Infection from the bone may spread to the costal cartilages, mediastinum, pleura, and pericardium with possible septicemia and multiorgan system failure. 
2. The associated patient's risk factors. Coronary disease, hypertension, diabetes mellitus, chronic obstructive pulmonary disease, obesity, and history of smoking are very common in such a group of patients. ${ }^{3}$

3. The poor vascular condition of the wound. The main blood supply of the anterior chest wall is frequently partially lost because 1 internal mammary artery has been used for myocardial revascularization.

The extent of the debridement is somewhat controversial. Resection of the entire sternum including the costochondral arches and the sternoclavicular joints (the so-called radical sternectomy) has been proposed. ${ }^{5}$ Radical sternectomy entails a more difficult reconstructive procedure, significant paradoxical chest movements, and a higher incidence of a postoperative clicking and discomfort. ${ }^{3}$ Furthermore, the site of the internal mammary artery harvest is usually more necrotic as compared with the contralateral, and frequently a wide debridement is required only on 1 side. For such reasons we think that radical sternectomy is to be considered appropriate only if required by the conditions of the wound. Currently, most patients are submitted to 1-stage debridement, and a primary open wound treatment should be reserved to those selected unstable patients with severe sepsis. ${ }^{3,6,7}$

The ideal flap for the reconstruction of anterior chest wall defects subsequent to sternal debridement has not yet been defined. Both omental and muscle flaps have proven to be effective reconstructive methods for the treatment of infected median sternotomy wounds, and the thoracic surgeon should adopt the technique with which he or she feels most familiar.

Muscle flaps are more commonly used because they are readily available. The surgical trauma is reduced. Most of the procedures are relatively quick and simple, and the results are generally good or acceptable. Pectoralis major based on the thoracoacromial vessels is considered the first choice by the majority of surgeons. Pectoralis major flaps based on the internal mammary perforants are less frequently used because in most patients these vessels have been used for myocardial revascularization or may be damaged because of infection.

Possible disadvantages of the pectoralis major flap are:

- It can be inadequate to fill the inferior third of the mediastinum $^{2,8-10}$

- It does not always provide sufficient bulk to fill wide defects, especially in female and in sicker patients. ${ }^{2}$

- It barely conforms to the deepest recesses of the mediastinum.

- Detachment of the muscle from its insertion on the humeral bone may cause functional sequelae. ${ }^{8}$

Other options include pedicled rectus abdominis muscle flap, pedicled latissimus dorsi flap, and free latissimus dorsi flap. All these procedures should be considered second choice for mediastinal reconstruction, basically because they are burdened by a substantial rate of local complications. $^{5}$

The use of the omentum is a valid alternative to the pectoralis major flaps. The favorable properties of the omentum are well known: it has a very rich blood supply and it is capable of inducing neovascularity and of surviving in highly contaminated fields, where it aids to eradicate the infection. ${ }^{4,11,12}$ Therefore, it has found applications in every surgical specialty, and its properties are appreciated also by those surgeons who commonly choose muscle flaps for the treatment of infected median sternotomy wounds. ${ }^{3,13}$ Its use is effective even in desperate situations such as in mediastinitis after cardiac transplantation or in sternal osteomyelitis after pneumonectomy. ${ }^{14,15}$ Lower mortality rates and fewer septic complications have been reported using the omental flap versus the pectoralis major flaps. ${ }^{2}$

The greatest disadvantage of the omentum for treating postoperative sternal osteomyelitis is linked to the laparotomy, which adds a considerable surgical trauma in already very sick patients. On the contrary, the risk of potential peritoneal contamination seems to be negligible. ${ }^{3}$ Laparotomy is responsible for postoperative pain that may interfere with the patient's ventilatory dynamic and may cause mucus retention, with possible respiratory infections. Furthermore, because of the postoperative ileus, it is more difficult to set the glucose values back to normal in diabetic patients.

Before developing our method of laparoscopic omentoplasty, we performed 10 open omental transpositions for the treatment of sternal osteomyelitis. We actually found that the omental flap, based on the gastroepiploic vessels, was generally too bulky for the defect following sternal debridement, especially at the lower third section of the wound. Volume reduction of the transposed omentum was frequently required to avoid heart compression or tension of the skin suture. Two patients with a very bulky omental flap, brought through the anterior rectus fascia, had a superior midline abdominal hernia that we did not repair for the risk of narrowing the vascular pedicle. Moreover, laparotomy and division of the left gastroepiploic vessels produced, in our series, an important oral nutrition delay, also secondary to the partial gastric wall devascularization.

Laparoscopic omental flap is a technically easy procedure if the division of gastroepiploic vessels is avoided. Such a procedure does not require any particular skill and could be carried out by any thoracic surgeon experienced in the laparoscopic treatment of gastroesophageal reflux disease. The only minor difficulty of the procedure is control of the correct position of the greater gastric curvature, which can be stretched cranially during the omental transposition. The hampered view of the gastric wall by the omental flap and the pneumoperitoneum leakage through the diaphragmatic incision could make this step fairly complex. 
A clinical and autopsy study demonstrated that simply detaching the omentum from the transverse colon produces a flap able to reach nipple level in $75 \%$ of patients. ${ }^{16}$ Further length is achievable by dividing some of the anastomotic vessels between the right gastroepiploic artery and Barkow's arcade within the flap. With such maneuvers the omentum can reach the base of the neck with a reasonable bulk reduction if compared with the omental flap based on the gastroepiploic vessels. On the other hand, division of the gastroepiploic vessels is mandatory only when the omental bulk appears too thin and/or for wider anterior chest-wall defect (eg, when the whole sternum and, bilaterally, all the cartilages have been resected). In such circumstances the laparoscopic procedure could be more difficult and the cooperation of a general surgeon, skilled in laparoscopic surgery, could be necessary.

The use of UltraCision Harmonic Scalpel is advisable, even though separation of the omentum from the transverse colon is mostly carried out by blunt dissection. The UltraCision Harmonic Scalpel makes the technique easier, without bleeding or smoke, and the release of possible omental adhesions simpler and safer.

Further experiences with the laparoscopic omentoplasty are needed. Theoretically for the treatment of sternal osteomyelitis, laparoscopic technique compared with the open procedure entails the following advantages:

1. The added surgical trauma is negligible.

2. Postoperative pain is lessened.

3. Postoperative bed rest is shorter.

4. Respiratory and wound complication rates are lower.

5. The possibility of theoretical peritoneal contamination is reduced.

6. Oral nutrition is resumed earlier.

7. It is easier to set the glucose values back to normal in diabetic patients.

8. Cosmetic results are improved.

\section{References}

1. Ottino G, De paulis R, Pansini S, et al. Major sternal wound infection after open heart surgery. A multivariate analysis of risk factors in 2579 consecutive operative procedures. Ann Thorac Surg. 1987;44:173-9.

2. Lopez-Monjardin H, de-la-Pena-Salcedo A, Mendoza-Munoz M, Lopez-Yanez-de-la-Pena A, Palacio-Lopez E, Lopez-Garcia A. Omentum flap versus pectoralis major flap in the treatment of mediastinitis. Plast Reconstr Surg. 1998;101:1481-5.

3. Jones G, Jurkiewicz MJ, Bostwick J, et al. Management of the infected median sternotomy wound with muscle flaps: the Emory 20-year experience. Ann Surg. 1997;225:766-78.

4. Mathisen DJ, Grillo HC, Vlahakes GJ, Daggett WM. The omentum in the management of complicated cardiothoracic problems. J Thorac Cardiovasc Surg. 1988;95:677-84.

5. Wettstein R, Erni D, Berdat P, Rothenfluh D, Banic A. Radical sternectomy and primary musculocutaneous flap reconstruction to control sternal osteitis. J Thorac Cardiovasc Surg. 2002;123:1185-90.

6. Schroeyers P, Wellens F, Degrieck I, et al. Aggressive primary treatment for poststernotomy acute mediastinitis: our experience with omentaland muscle flap surgery. Eur J Cardiothorac Surg. 2001;20:743-6.

7. Totaro P, Borghetti V, Lanzi S, Minzioni G. Primary omental-flap surgery for post-sternotomy acute mediastinitis. Eur J Cardiothorac Surg. 2002;21:765.

8. Iacobucci JJ, Stevenson TR, Hall JD, Deeb GM. Sternal osteomyelitis: treatment with rectus abdominis muscle. Br J Plast Surg. 1989;42: 452-9.

9. Mathes SJ. The principles of muscle and musculocutaneous flaps. In: McCarthy JG, editor. Plastic surgery. Vol 1. Philadelphia: W. B. Saunders; 1990: chap 11.

10. Georgiade G. Mediastinitis. In: Georgiade G, editor. Textbook of plastic, maxillofacial and reconstructive surgery. Vol. 2, 2nd ed. Baltimore: Williams and Wilkins; 1992. p. 883-91.

11. Goldsmith HS, Griffith AL, Kupferman A, Catsimpoolas N. Lipid angiogenic factor from omentum. JAMA. 1984;252:2034-6.

12. Omura K, Misaki T, Takahashi H, Kobayashi K, Watanabe Y. Omental transfer for the treatment of sternal infection after cardiac surgery: report of three cases. Surg Today. 1994;24:67-71.

13. Pairolero PC, Arnold PG. Management of recalcitrant median sternotomy wounds. J Thorac Cardiovasc Surg. 1984;88:357-64.

14. Wornom IL 3rd, Maragh H, Pozez A, Guerraty AJ. Use of the omentum in the management of sternal wound infection after cardiac transplantation. Plast Reconstr Surg. 1995;95:697-702.

15. Miwa K, Takamori S, Mitsuoka M, Hayashi A, Fukunaga M, Shirouzu K. Successful treatment of sternal osteomyelitis after pneumonectomy using a pedicled omental flap. Jpn J Thorac Cardiovasc Surg. 2001;49:522-4.

16. Das SK. The size of human omentum and methods of lengthening it for transplantation. Br J Plast Surg. 1976;29:170-4. 